\title{
Analysis of the non-isothermal Newtonian model in the blown film process
}

\author{
J. C. Bennett* J. J. Shepherd ${ }^{\dagger}$
}

(Received 11 December 2005; revised 21 August 2006)

\begin{abstract}
Film blowing is an industrial process used to produce thin sheets of polymer with a wide range of applications; for example, plastic bags. We apply analytic and numerical techniques to model this process in the simplest case where the blown material is a Newtonian fluid operating under non-isothermal conditions. In particular, we obtain the thermal profile along the film, and the effects of this thermal variation on the film bubble radius.
\end{abstract}

\section{Contents}

\section{Introduction}

*School of Mathematical and Geospatial Sciences, Royal Melbourne Institute of Technology, Melbourne, Australia. mailto:E58144@ems.rmit.edu.au

${ }^{\dagger}$ School of Mathematical and Geospatial Sciences, Royal Melbourne Institute of Technology, Melbourne, Australia. mailto: jshep@rmit.edu.au

See http://anziamj . austms .org.au/V47EMAC2005/Bennett for this article, (c) Austral. Mathematical Soc. 2006. Published October 2, 2006. ISSN 1446-8735 
2 Non-dimensional governing equations

3 Analytic approximation

C328

4 Linearization and the iterative scheme

C331

5 Results and discussion

C332

References

C337

\section{Introduction}

The industrial process of film blowing, by which thin polymer film is manufactured from molten polymer has been studied extensively for a wide range of polymeric materials. The mathematical models used to represent film blowing involve highly nonlinear differential equations that describe the complex features of the process. This complexity leads to the analysis of these being almost totally numerical. A good survey of the models involved and their analysis may be found in [4].

Shepherd \& Bennett [5] considered the simplest film blowing case - that of a Newtonian film being blown under isothermal conditions. There, a mixture of singular perturbation and other heuristic techniques were applied to construct a leading order approximation to the film bubble profile over the extent of the blowing process. Here we extend these techniques to include temperature variation in the Newtonian film. The (isothermal) analytical approximation obtained in [5] is used as an initial approximate solution to the thermal model, then the nonlinear thermal differential equation governing the film radius is linearized around this approximate solution and a solution to the full thermal equations obtained using iteration. The thermal profile along the film bubble is constructed as a side calculation. 
The Newtonian model analyzed here is, to some extent, a simplification of the real-world situation where molten polymer is used to manufacture plastic film. However, it is sufficiently complex to provide most of the film bubble structure found in more realistic and complicated models.

\section{Non-dimensional governing equations}

The essential features of the geometry and organization of the film blowing process are described in $[1,5,6,7]$ for isothermal Newtonian films. Put simply, molten polymer is extruded through a narrow annular die, with the resulting tubular film "bubble" being drawn upwards, its shape being maintained by an applied internal air pressure. At a particular height above the die (the "freeze line"), the polymer solidifies and is drawn off between pinch rollers as a double plastic sheet.

For the present case of a Newtonian film with thermal variation, these details remain essentially the same, with the added feature of a temperature distribution along the film bubble, together with equations governing that distribution. If gravity effects are neglected, and the film bubble is assumed to be axially symmetric, the bubble geometry and its thermal state may be described by two dimensionless state variables, namely the dimensionless film radius and temperature, $r(z)$ and $s(z)$ respectively. Here, $z$ is the dimensionless distance along the film axis, scaled against the freeze line distance - thus, $0 \leq z \leq 1$; and $r(z)$ and $s(z)$ are scaled against the die exit radius and die exit temperature respectively. Application of mechanical and thermal balance laws then lead to $r(z)$ and $s(z)$ being determined by the differential equations

$$
\begin{aligned}
& 2 C^{2} r^{2}\left[f_{0}+B\left(r^{2}-1\right)\right] r^{\prime \prime} \\
& -6 C \eta r^{\prime}-r\left[f_{0}-B\left(3 r^{2}+1\right)\right]\left[1+C^{2}\left(r^{\prime}\right)^{2}\right]=0, \\
& s^{\prime}+H r \sqrt{1+C^{2}\left(r^{\prime}\right)^{2}}\left(s-s_{a}\right)+J r \sqrt{1+C^{2}\left(r^{\prime}\right)^{2}}\left(s^{4}-s_{a}^{4}\right)=0,
\end{aligned}
$$


subject to the conditions

$$
\begin{aligned}
& r(0)=1, \quad r^{\prime}(1)=0, \\
& s(0)=1 .
\end{aligned}
$$

In the above, the dimensionless parameters $B, C$ and $f_{0}$ are as defined in [5], and are dimensionless versions of the applied internal bubble pressure, the bubble "aspect ratio", that is, ratio of die exit radius to freezeline distance, and roller pulling force, respectively. Temperature effects in the film radius equation (1) occur only in the Arrhenius factor

$$
\eta(z)=\exp \left[w\left(\frac{1}{s}-1\right)\right] .
$$

The dimensionless parameters $H, J, w$ and $s_{a}$ occurring in the temperature equation (2) and $\eta(z)$ relate to thermal effects, with $H$ a scaled heat transfer coefficient, $J$ a scaled emissivity, $w$ a scaled activation energy and $s_{a}$ a scaled ambient temperature.

We recover the isothermal case considered in [5] from the above equations by setting $s(z) \equiv 1$ ( that is, the die exit temperature) and consequently, $\eta(z) \equiv 1$.

\section{Analytic approximation}

As mentioned in the Introduction, our earlier work $[1,5]$ (extending [6]) used a mixed approach to construct an approximate expression for the film bubble profile in the the isothermal case, based on the assumption of small values of the parameter $C$. Earlier numerical studies [1] indicated that as $C \rightarrow 0$, the bubble profile $r(z)$ displayed an interior layer structure (that is, region of rapid change) centred on a point $z=a$ interior to $0 \leq z \leq 1$. To construct a suitable approximation to the bubble radius profile $r(z)$, we divided 
this interval into two subintervals, $0 \leq z \leq a$ and $a \leq z \leq 1$; and the condition $r(a)=\lambda$ was imposed. Thus, $a$ and $\lambda$ became unknown quantities to be determined. On the first interval, we solved the governing differential equation (namely, (1), with $\eta \equiv 1$, for the isothermal case) approximately, using the method of matched expansions, to meet the first of (3) and the condition $r(a)=\lambda$. On the second, a suitable approximating equation to (1) was constructed and solved to satisfy the condition at $z=a$ and the second of (3). The values of $a$ and of $\lambda$ were then determined by requiring that the expressions on the two subintervals met smoothly at $z=a$. Note that the approximating equation on the second subinterval required inclusion of $\rho_{\mathrm{BU}}$, the blow up ratio, or ratio of film bubble radius at the freezeline to that at the die exit. Thus, we assume $\rho_{\mathrm{BU}}$ to be a known quantity (as is the case in practical applications). Finally, if $r_{1}(z)$ and $r_{2}(z)$ designate the approximations to the bubble radius $r(z)$ on the first and second subintervals respectively, we represent the "composite" approximation over the whole interval $0 \leq z \leq 1$, $r_{c}(z)[5]$ as

$$
r_{c}(z)=r_{1}(z)(H(z)-H(z-a))+r_{2}(z)(H(z-a)-H(z-1)) .
$$

Here, $H(z)$ is the Heaviside function, and

$$
\begin{aligned}
r_{1}(z, C)= & \phi(z, C)+(\phi(a, C)-\lambda) \operatorname{erf}\left[\frac{\sqrt{2 M}(a-z)}{2 C}\right] \\
& +\lambda-\phi(a, C), \\
\text { with } \quad & \phi(z, C)=\left\{\left(1-\frac{3 B}{f_{0}-B}\right) \exp \left[\left(\frac{f_{0}-B}{3 C}\right) z\right]+\frac{3 B}{f_{0}-B}\right\} \\
\text { and } \quad & r_{2}(z, C)=\lambda+\frac{1}{18} \rho_{\mathrm{BU}}^{3} \sigma \gamma\left(e^{\nu(z, C)}-e^{\nu(a, C)}\right)-\frac{\rho_{\mathrm{BU}}}{6 C} \gamma(z-a), \\
\text { where } \quad & \sigma=\left[f_{0}+B\left(\rho_{\mathrm{BU}}^{2}-1\right)\right] \\
& \gamma=\left[f_{0}-B\left(3 \rho_{\mathrm{BU}}^{2}+1\right)\right] \\
\text { while } & \nu(z, C)=\frac{3(z-1)}{C \sigma \rho_{\mathrm{BU}}^{2}} .
\end{aligned}
$$$$
\text { with } \quad \phi(z, C)=\left\{\left(1-\frac{3 B}{f_{0}-B}\right) \exp \left[\left(\frac{f_{0}-B}{3 C}\right) z\right]+\frac{3 B}{f_{0}-B}\right\}^{-1 / 2}(8)
$$ 


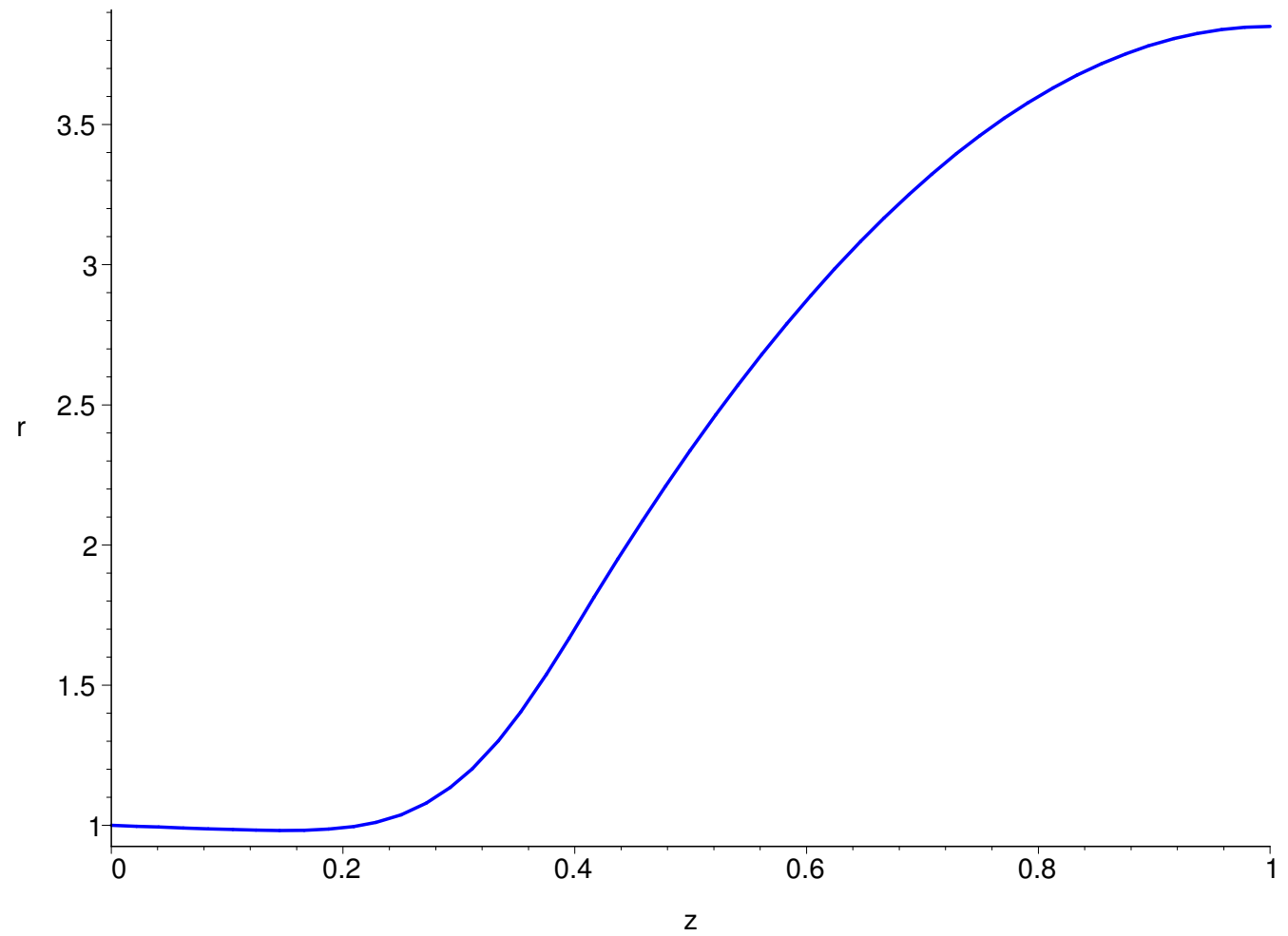

FiguRE 1: Typical approximate isothermal bubble radius profile $r_{c}(z)$.

Figure 1 shows a plot of the isothermal approximation $r_{c}(z)$ for parameter values $B=0.21, C=0.15, f_{0}=0.969, \rho_{\mathrm{BU}}=3.85$. These correspond to $a \simeq 0.41, \lambda \simeq 1.77$. The interior layer structure centred (approximately) on $z=a$ is clearly apparent. Note that, on leaving the die exit, the polymer tube radius reduces slightly, before expanding to the eventual value of $\rho_{\mathrm{BU}}$ at the freezeline. This phenomenon, known as necking, occurs for various values of the film parameters, and, significantly, the sharpening layer structure noted above only occurs when necking is present. 


\section{Linearization and the iterative scheme}

The differential equations $(1,2)$ comprise a highly nonlinear system, with the only coupling occurring via the factor $\eta(z)$. Thus, for a given function $s(z)$, Equation (1) together with the boundary conditions (3) comprise a nonlinear two point boundary value problem, that may be solved iteratively, for an appropriate initial iterate. The resulting profile $r(z)$ could then be substituted into Equation (2); and the resulting equation solved subject to (4), to give an approximate temperature profile $s(z)$. This "see-saw" process might be continued, to give, assuming convergence, the film and temperature profiles in the bubble. This is the approach we use here, with our initial iterate the approximate isothermal profile $r_{c}(z)$ of the previous section.

To recast Equation (1) in a form for iterative solution, we write the (unknown) solution $r(z)$ as

$$
r(z)=R(z)+u(z),
$$

where $R(z)$ is a suitably chosen known function. Substituting (13) into Equation (1), expanding, and collecting the linear terms in $u(z)$ whilst holding $\eta(z)$ fixed, yields the following equation for the unknown $u(z)$ :

$$
a(R) u^{\prime \prime}+b(R, s) u^{\prime}+c(R) u=N(R, s)+Q\left(R, u, u^{\prime}\right) .
$$

In the above, $N(R, s)$ is the residual, that is, the result of direct substitution $r=R$ into the left-hand side of Equation (1). Note that it includes the (assumed given) function $s(z) . Q\left(R, u, u^{\prime}\right)$ is a nonlinear term, at least quadratic in $u$ and $u^{\prime}$, which does not include $s(z)$.

We now use Equation (14) to define a map from $\tilde{u}$ onto $u$, for given $R(z)$ and $s(z)$, as the solution of the linear nonhomogeneous differential equation

$$
a(R) u^{\prime \prime}+b(R, s) u^{\prime}+c(R) u=N(R, s)+Q\left(R, \tilde{u}, \tilde{u}^{\prime}\right),
$$


subject to the boundary conditions

$$
u(0)=0, \quad u^{\prime}(1)=0 .
$$

We now set up our iterative procedure as follows. We begin by choosing our $R(z)$ above to be the $r_{c}(z)$ of the previous section. We then calculate an initial (approximate) temperature profile $s(z)$ by solving $(2,4)$ with the estimate $r(z)=r_{c}(z)$. With this $s(z)$ and the above $R(z)$, we solve the linear boundary value problem $(15,16)$ with $\tilde{u}=0$. The resulting $u(z)$ is then applied to (13) to give an updated $r(z)$, which is then applied to $(2$, $4)$, and an updated $s(z)$ is obtained. The problem $(15,16)$ is now re-solved, with $\tilde{u}$ on the right hand side of (15) replaced with our updated $u$, and our updated $s(z)$ applied throughout. This gives a new $u$, hence a new $r$ via (13), and so the iterative process continues. Note that we have the option to use the updated $r(z)$ as the $R(z)$ in (14) at any stage (or all stages) of the iteration process. This can be used to improve convergence.

The approach described above has some desirable features. The usual way to solve the above problem for $r(z)$ and $s(z)$ has been to solve as an initial value problem, using a shooting method. The layer structure in $r_{c}(z)$ for small $C$ seen above is also reflected in the actual solution $r$, and this has meant that instabilities are often encountered using this method. However, iteration based on $r_{c}(z)$ means that we are solving for small iterates $u$, which have, at worst, a very limited layer structure, unlikely to cause instability. This is found to be the case.

\section{$5 \quad$ Results and discussion}

The iterative numerical technique described above was employed, with our choice of values for the parameters $B, C, f_{0}, \rho_{\mathrm{BU}}$ as above; while values for the thermal parameters were $H=0.01, J=0.03, w=7.448$ and $s_{a}=0.1$. Solution of the two point linear boundary value problem $(15,16)$ and construction 
TABLE 1: maximum values of the difference of successive iterates.

\begin{tabular}{ccl} 
Stage & $n$ & $\left|u_{n+1}-u_{n}\right|_{\max }$ \\
\hline 1 & 1 & 0.188 \\
1 & 2 & 0.122 \\
1 & 3 & \\
2 & 4 & 0.001649 \\
2 & 5 & 0.000210 \\
2 & 6 & \\
3 & 7 & 0.000166 \\
3 & 8 & 0.000000332 \\
3 & 9 & \\
\hline
\end{tabular}

of the temperature profile $s(z)$ from the nonlinear initial value problem $(2$, 4) was carried out at each stage using Maple's default numerical solvers. We found that computation was significantly accelerated by representing $r_{c}(z)$ by a quintic spline approximation where it occurred (as $R$ ) in the coefficients of (15) and (2). The iteration process converged satisfactorily within nine steps; and this is indicated in Table 1, where successive iterates obtained by solving (15) are displayed. (The gaps in the table indicate iteration stages at which $R(z)$ in (14) was updated, as described above).

The resulting bubble radius and temperature profiles $r(z)$ and $s(z)$ respectively are given in Figures 2 and 3 respectively. We see that necking is present (as in the isothermal approximation, above), unchanged by the presence of the thermal profile (at least for the parameter values used here). Further, the value of the blow up ratio, $\rho_{\mathrm{BU}}$ (that is, $r(1)$ ) has increased noticeably relative to that for $r_{c}(z)$ above. We can see this more clearly in Figure 4, where the thermal profile and isothermal approximation $r_{c}(z)$ are displayed.

The temperature profile also shows a monotonic decrease along the film (overall, about $10 \%$ here), together with a (relatively) rapid slope change at $z \simeq 0.41-$ a consequence of the radius profile layer there. 


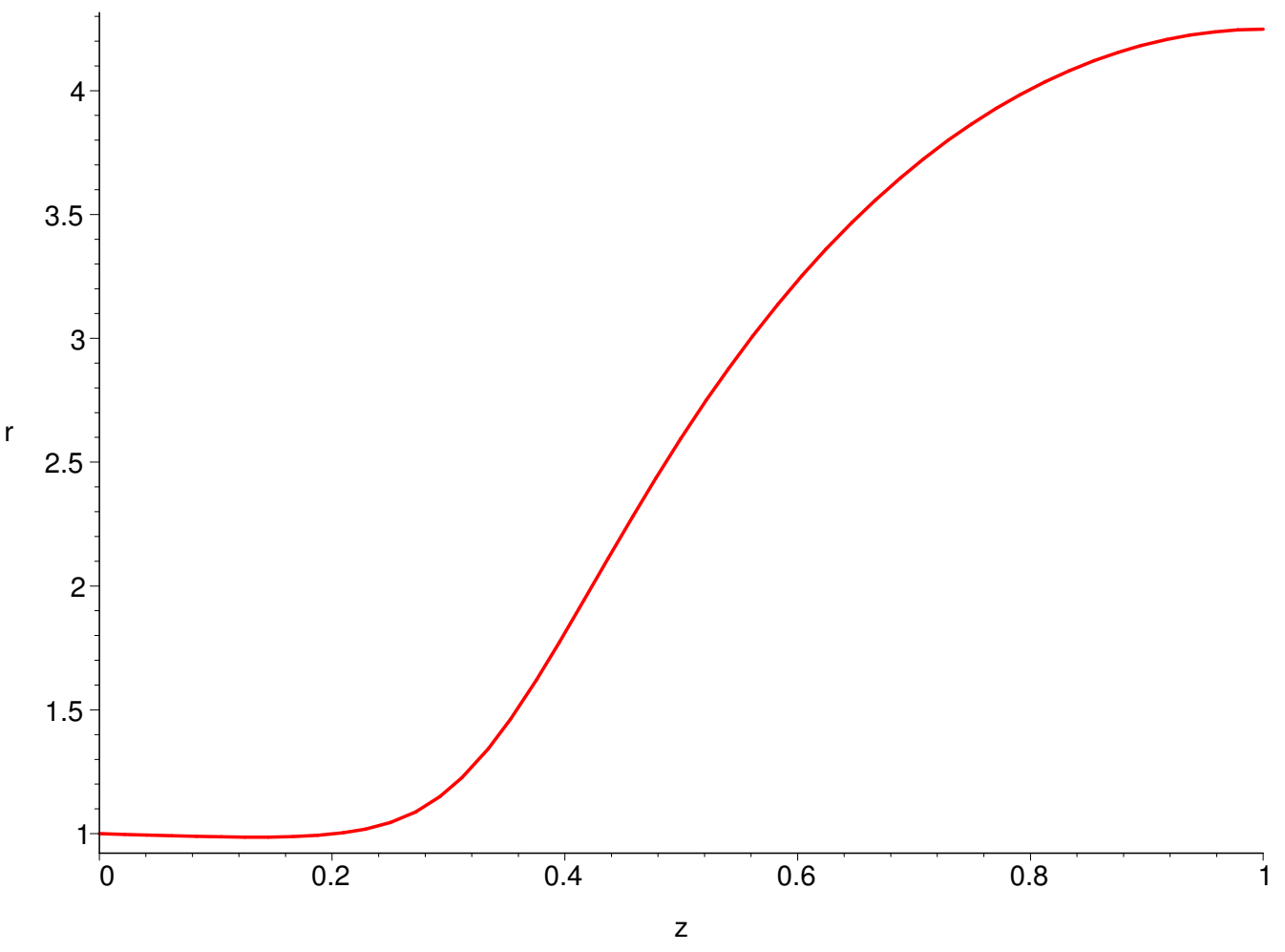

Figure 2: Thermal bubble radius profile $r(z)$. 


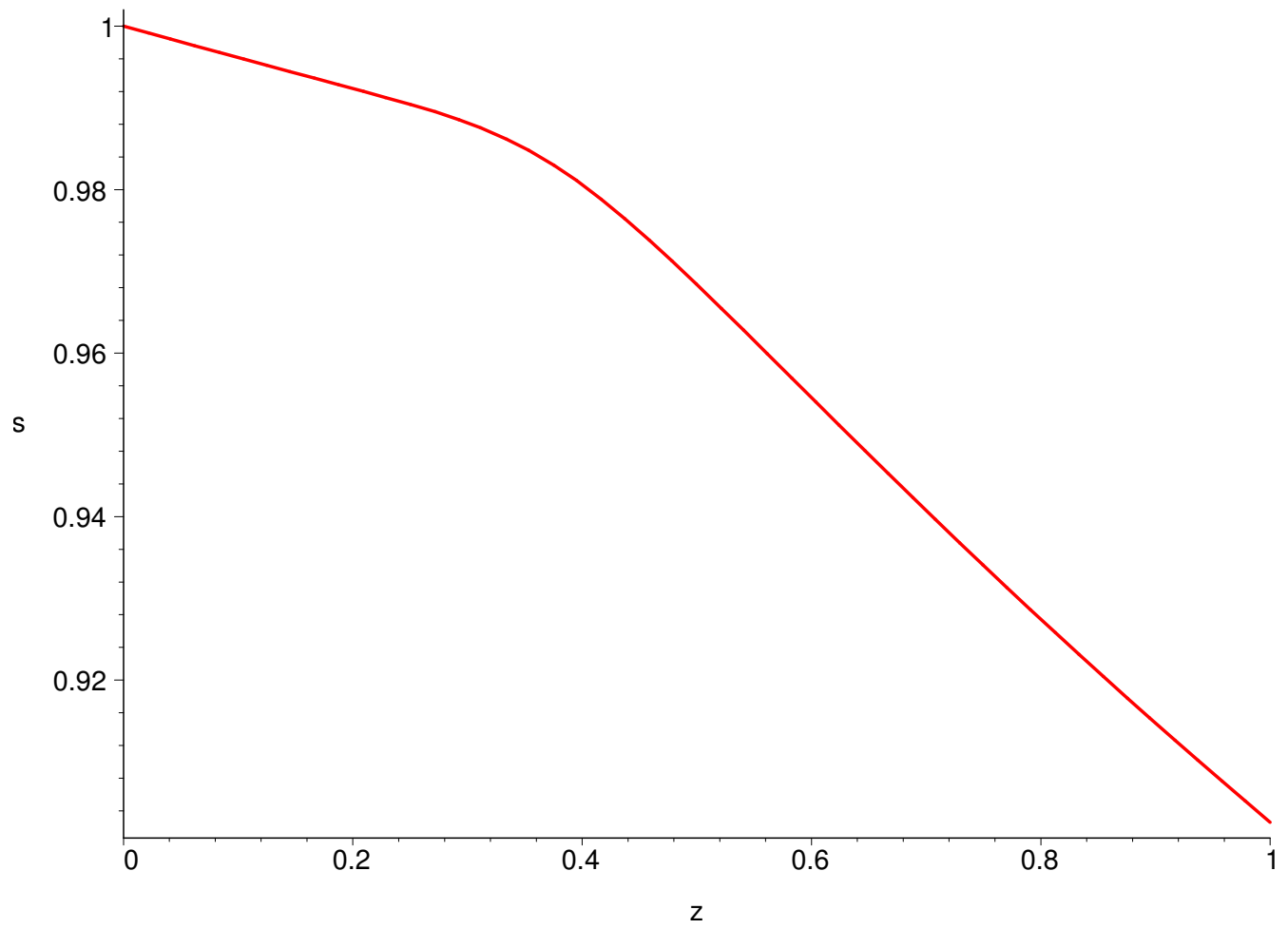

Figure 3: Bubble temperature profile $s(z)$. 

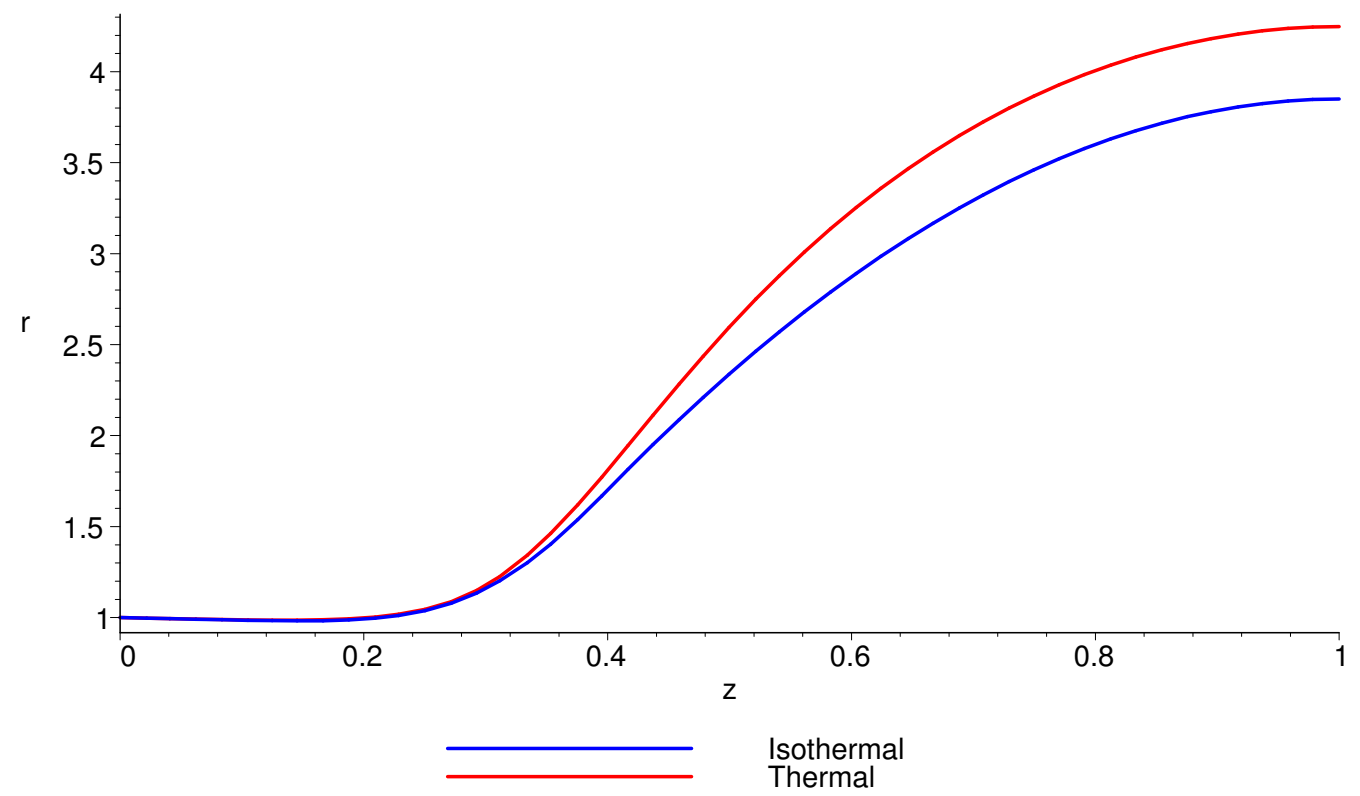

Figure 4: Comparison between thermal and approximate isothermal bubble radius profiles. 
When there is no necking a different situation arises. The layer structure on which our calculation of $r_{c}(z)$ is based is greatly reduced or absent. The gradient of profiles in the transition region for this set becomes shallower in the limit as $C \rightarrow 0$. Interestingly, in many such cases the approximate solution $r_{c}(z)$ agrees quite well with the numerical solution [1]. Under these conditions multiple solutions may occur $[3,2]$.

\section{References}

[1] Bennett, J. C., Interior Layer Structure in the Blown Newtonian Film, Honours Thesis, School of Mathematical and Geospatial Sciences, RMIT University, 2004. C327, C328, C337

[2] Cain, J. J. and Denn, M. M., Multiplicities and instabilities in film blowing, Polymer Engineering and Science, 28, 1988, pp.1527-1541. C337

[3] Ervin, V. J., Non-uniqueness of the Newtonian film blowing model, in preparation. C337

[4] Muke, S., Connell, H., Sbarski, I. and Bhattacharya, S. N., Numerical modelling and experimental verification of blown film processing, Journal of Non-Newtonian Fluid Mechanics, 116, 2003, pp.113-138. http://dx.doi.org/10.1016/j.jnnfm.2003.09.002 C326

[5] Shepherd, J. J. and Bennett, J. C., Interior layer structure in the Newtonian blown film, ANZIAM Journal 46(E), 2005, pp.C101-C116. http://anziamj . austms.org.au/V46/CTAC2004/Shep/ C326, C327, C328, C329

[6] Shepherd, J. J.,Connell, H. J. and Tam, D. C. H., An interior layer in a film-blowing problem, Proceedings of the Fifth Biennial Engineering Mathematics and Applications Conference (EMAC2002), 
M. Pemberton, I. Turner, P. Jacobs (eds), The Institution of Engineers Australia, Brisbane, Queensland (2002), pp.181-186. C327, C328

[7] Tam, D. C. H., Shepherd, J. J. and Connell, H. J., Modelling the film blowing process, Proceedings of the Fourth Biennial Engineering Mathematics and Applications Conference (EMAC2000), R. L. May, G. F. Fitz-Gerald, I. H. Grundy (eds), The Institution of Engineers Australia, Melbourne (2000), pp 271-274. C327 